\title{
INDEX THEORY FOR TOEPLITZ OPERATORS ON BOUNDED SYMMETRIC DOMAINS
}

\author{
HARALD UPMEIER
}

In this note we give an index theory for Toeplitz operators on the Hardy space of the Shilov boundary of an arbitrary bounded symmetric domain. Our results generalize earlier work of Gohberg-Krein and Venugopalkrishna [12] for domains of rank 1 and of Berger-Coburn-Korányi [1] for domains of rank 2.

Bounded symmetric domains (Cartan domains, classical or exceptional) are the natural higher-dimensional analogues of the open unit disk. Each such domain is homogeneous under a semisimple Lie group of biholomorphic transformations and has an (essentially unique) realization as a convex circular domain which is conveniently described in Jordan algebraic terms: The underlying vector space $Z \approx \mathbf{C}^{n}$ carries a Jordan triple product, denoted by $(u, v, w) \mapsto\left\{u v^{*} w\right\}$, and the bounded symmetric domain $D$ is the open unit ball of $Z$ with respect to the "spectral norm" [7]. The basic example is the space $Z=\mathbf{C}^{p \times q}$ of rectangular matrices, with Jordan triple product $\left\{u v^{*} w\right\}:=\left(u v^{*} w+w v^{*} u\right) / 2$, giving rise to the "hyperbolic matrix ball" $D=\left\{z \in Z\right.$ : spectrum $\left.\left(z^{*} z\right)<1\right\}$.

The symmetric domains of rank 1 are the (smooth) Hilbert balls in $\mathbf{C}^{n}$. For domains $D$ of higher rank $r$, the boundary $\partial D$ forms a nonsmooth "stratified space". In Jordan algebraic terms, the structure of $\partial D$ can be described as follows: An element $e \in Z$ satisfying $\left\{e e^{*} e\right\}=e$ is called a tripotent. For matrices, the tripotents are just the partial isometries. Every tripotent $e$ induces a splitting of $Z$ into the "Peirce spaces" $Z_{\lambda}(e):=\left\{z \in Z:\left\{e e^{*} z\right\}=\right.$ $\lambda z\}$ for $\lambda=0, \frac{1}{2}, 1$. The set

$$
D^{e}:=D \cap Z_{0}(e)
$$

is a bounded symmetric domain in $Z_{0}(e)$ and, by [7], the translated sets $e+D^{e}$, for nonzero tripotents $e$, constitute all boundary components ("faces") of $D$. For $j \leq r$, these components can be organized into smooth families, labeled by the compact manifold $S_{j}$ of all tripotents of equal rank $j$. The Shilov boundary $S$ of $D$ coincides with $S_{r}$ [7]. $S$ is homogeneous under the connected linear automorphism group $K$ of $D$ and thus carries a $K$-invariant probability measure. For irreducible $D$, the same is true of the "partial Shilov boundaries" $S_{j}$. We assume in the following that $D$ is irreducible.

The Hardy space $H^{2}(S)$ over $S$ and the dense subspace $P(Z)$ of all polynomials have been analyzed by W. Schmid [8], who showed that $P(Z)$ has an iso-

Received by the editors April 28, 1986 and, in revised form, August 26, 1986.

1980 Mathematics Subject Classification (1985 Revision). Primary 47B35, 58G10; Secondary 32M15, $17 \mathrm{C} 35$.

Supported in part by NSF grant DMS-8403631. 
typic decomposition into inequivalent irreducible $K$-modules $E_{m}$, where $m=$ $\left(m_{1}, \ldots, m_{r}\right)$ describes all "signatures" of integers $m_{1} \geq m_{2} \geq \cdots \geq m_{r} \geq 0$. For $j<r$, let $P_{j}(Z)$ be the sum of all $E_{m}$ with $m=\left(m_{1}, \ldots, m_{j}, 0, \ldots, 0\right)$. Denote by $H^{2}\left(S_{j}\right)$ the closed subspace of $L^{2}\left(S_{j}\right)$ defined by the tangential Cauchy-Riemann equations on $S_{j}$. Modifying the proof in [8], we get

THEOREM 1. The restriction mapping $P_{j}(Z) \rightarrow H^{2}\left(S_{j}\right)$ is injective and dense, and the isotypic components $E_{\left(m_{1}, \ldots, m_{j}, 0, \ldots, 0\right)}$ of $H^{2}\left(S_{j}\right)$ occur only once in $L^{2}\left(S_{j}\right)$.

By definition, the Toeplitz operator $T(S) f$ with continuous "symbol function" $f \in C(S)$ maps $h \in H^{2}(S)$ to $\pi_{S}(f h)$, where $\pi_{S}: L^{2}(S) \rightarrow H^{2}(S)$ is the orthogonal (Szegö) projection. By [10], the Toeplitz $C^{*}$-algebra $\tau_{S}$ generated by these operators has a composition series

$$
\{0\}=: I_{0} \subset I_{1} \subset \cdots \subset I_{r} \subset I_{r+1}:=\tau_{S}
$$

of length $r=\operatorname{rank}(D)$, with subquotients $I_{j+1} / I_{j} \approx C\left(S_{j}\right) \otimes K(j<r)$ and $I_{r+1} / I_{r} \approx C(S)$. Here $K$ denotes the $C^{*}$-algebra of all compact operators. In particular, the spectrum of $\tau_{S}$ is precisely the set of all tripotents. For a tripotent $e$, the corresponding irreducible representation $\sigma^{e}$ of $\tau_{S}$ acts on the Hardy space $H^{2}\left(S^{e}\right)$ of the Shilov boundary $S^{e}$ of the boundary component $D^{e}\left(\mathrm{cf}\right.$. (1)) and is uniquely determined by the property $\sigma^{e}(T(S) f)=T\left(S^{e}\right) f^{e}$ for all $f \in C(S)$. Here we put

$$
f^{e}(w):=f(e+w)
$$

for all $w \in S^{e}$. The product representation $\sigma_{j}:=\left\{\sigma^{e}: e \in S_{j}\right\}$ has the null-space $I_{j}$ and induces the subquotient isomorphism mentioned above.

For $1 \leq j \leq r,(2)$ induces a $C^{*}$-algebra extension

$$
0 \rightarrow C\left(S_{j-1}\right) \otimes K \rightarrow I_{j+1} / I_{j-1} \stackrel{\sigma_{j}}{\rightarrow} C\left(S_{j}\right) \otimes K \rightarrow 0,
$$

called the $j$ th Toeplitz extension over $S$ and denoted by $\tau_{j}(S)$. Let $\tau_{j}$ be any completely positive cross-section of $\sigma_{j}$ (cf. [4]) and, using $K$-theoretic notation, define the analytical $j$-index

$$
\operatorname{Ind}_{j}: K^{1}\left(S_{j}\right) \rightarrow K^{0}\left(S_{j-1}\right)
$$

by putting $\operatorname{Ind}_{j}(\alpha):=\operatorname{Index}\left(\sigma_{j-1}\left(\tau_{j}(\alpha)\right)\right.$ for every $\alpha \in K^{1}\left(S_{j}\right) \approx\left[S_{j}, G L_{\infty}\right]$. Note that $\tau_{j}(\alpha) \in \tau_{S}$ is invertible modulo $I_{j}$ and hence $\sigma_{j-1}\left(\tau_{j}(\alpha)\right)$ is a continuous family of Fredholm operators over $S_{j-1}$ (cf. [5]). For $j=1$, we get the usual integer-valued Fredholm index.

In order to describe (5) topologically, we compare (4) with a Toeplitz extension studied in [3]. The space $S_{1}$ of all minimal tripotents is a strictly pseudoconvex boundary in the singular analytic cone $\{z \in Z: \operatorname{rank}(z) \leq 1\}$. We can therefore consider "Boutet de Monvel type" Toeplitz operators $T\left(S_{1}\right) f$ on $H^{2}\left(S_{1}\right)$ (cf. Theorem 1), with symbol $f \in C\left(S_{1}\right)$. Since $S_{1}$ is strictly pseudoconvex, these operators have compact commutators and thus define an extension of $\mathcal{K}$ by $C\left(S_{1}\right)$, denoted by $\tau\left(S_{1}\right)$. For $j>1$, we apply this construction to the spaces $S_{1}^{e}:=S_{1} \cap Z_{0}(e)$, for $e \in S_{j-1}$, and obtain the Toeplitz extension $\tau\left(S_{j}\right)$ over $S_{j}$ defined via its "Busby invariant" (cf. (3))

$$
C\left(S_{j}\right) \ni f \mapsto\left(T\left(S_{1}^{e}\right) f^{e}\right)_{e \in S_{j-1}} .
$$


Our main analytical result $[\mathbf{1 1}]$ is the following.

THEOREM 2. The extensions $\tau_{j}(S)$ and $\tau\left(S_{j}\right)(c f .(4)$ and $(6)$ ) are stably (unitarily) equivalent, hence give rise to the same element in the Kasparov group $\operatorname{Ext}\left(C\left(S_{j}\right), C\left(S_{j-1}\right)\right)$.

The main difficulty in the proof lies in the fact that the extensions $\tau_{j}(S)$ and $\tau\left(S_{j}\right)$ "live" on different measure spaces $S$ and $S_{j}$. First consider the case $j=1$, giving the usual index. By Theorem 1 , there exist constants $\lambda_{m}, m \in$ $\mathbf{N}$, such that the mapping $U f:=\lambda_{m} \cdot f \mid S_{1}$ is an isometry from $E_{(m, 0, \ldots, 0)} \subset$ $H^{2}(S)$ into $H^{2}\left(S_{1}\right)$. It follows that $U$ extends to a unitary mapping between the closure $H_{1}^{2}(S)$ of $P_{1}(Z)$ and $H^{2}\left(S_{1}\right)$. Let $N_{m}$ be the highest weight vector in $E_{(m, 0, \ldots, 0)}$. Then the explicit calculations of [9, Theorem 2.1 and Lemmas 2.6 and 2.7] imply that the quotients $\Lambda_{m}:=\left(N_{m} \mid N_{m}\right)_{S} /\left(N_{m} \mid N_{m}\right)_{S_{1}}$ of the respective $L^{2}$-scalar products satisfy $\Lambda_{m} / \Lambda_{m+1} \rightarrow 1$ as $m \rightarrow \infty$. This implies that $U$ induces a unitary equivalence (cf. $[2,6]$ ) between $\tau_{1}(S)$ (compressed to $\left.H_{1}^{2}(S)\right)$ and $\tau\left(S_{1}\right)$. For $j>1$, one applies these arguments to the boundary components $D^{e}$, for $e \in S_{j-1}$, and shows that the operator fields inducing the unitary equivalence are continuous in the parameter $e$.

By Theorem 2, we can use the index formula for (families of) Toeplitz operators on strongly pseudoconvex (singular) domains $[3$, Theorem 1 and Final Remarks] to express (5) in topological terms:

THEOREM 3. For $j=1$, the 1-index $\operatorname{Ind}_{1}: K^{1}\left(S_{1}\right) \rightarrow \mathbf{Z}$ is the index character [3] of the oriented contact manifold $S_{1}$ and has the cohomological expression

$$
\operatorname{Ind}_{1}(\alpha)=\operatorname{ch}(\alpha) \cdot \operatorname{Td}(E)\left[S_{1}\right]=\sum_{k \geq 1} \frac{-1}{(k-1) !} \alpha^{*} h_{k} \cdot \operatorname{Td}(E)\left[S_{1}\right]
$$

for all $\alpha \in\left[S_{1}, G L_{\infty}\right]=\left[S_{1}, U_{\infty}\right]$. Here ch denotes the (odd) Chern character $[0,1.10], \operatorname{Td}(E)$ is the Todd class of the "Peirce $\frac{1}{2}$-bundle" $E=\left(Z_{1 / 2}(e)\right)$ over $S_{1}$ and $h_{1}, \ldots, h_{n}$ are the standard generators of $H^{*}\left(U_{n}, \mathbf{Q}\right)$.

Similarly, the higher indices $\operatorname{Ind}_{j}$ are given by $\operatorname{Ind}_{j}(\alpha)=\chi(\beta \alpha)$ for all $\alpha \in K^{1}\left(S_{j}\right)$, where $\chi$ is the index character of the fibre bundle $\pi_{j}: \Sigma_{j} \rightarrow S_{j-1}$ with fibres $S_{1}^{e}, e \in S_{j-1}$, and $\beta: \Sigma_{j} \rightarrow S_{j}$ maps $c \in S_{1}^{e}$ to $e+c$. The cohomological expression for $\operatorname{Ind}_{j}(\alpha)$ is similar to (7) involving integration along the fibres of $\pi_{j}$. Geometrically, $\beta$ realizes a boundary component of $D^{e}$ as a boundary component of $D$ itself. For domains of rank 1 (Hilbert balls) and rank 2 ("Lie balls"), Theorem 3 gives the "odd" index theorem for Toeplitz operators [12] and Atiyah-Singer's "even" index theorem over spheres [1], respectively. Theorem 3 can also be applied to deduce index formulas for Wiener-Hopf operators on (irreducible) self-dual homogeneous cones.

\section{REFERENCES}

0. M. F. Atiyah and F. Hirzebruch, Vector bundles and homogeneous spaces, Proc. Sympos Pure Math., vol. 3, Amer. Math. Soc., Providence, R. I., 1961, pp. 7-38.

1. C. A. Berger, L. A. Coburn and A. Korányi, Opérateurs de Wiener-Hopf sur les sphères de Lie, C. R. Acad. Sci. Paris 290 (1980), 989-991. 
2. L. G. Brown, R. G. Douglas and P. A. Fillmore, Extensions of $C^{*}$-algebras and K-homology, Ann. of Math. 105 (1977), 265-324.

3. L. Boutet de Monvel, On the index of Toeplitz operators of several complex variables, Invent. Math. 50 (1979), 249-272.

4. M. D. Choi and E. G. Effros, The completely positive lifting problem, Ann. of Math. (2) 104 (1976), 585-609.

5. A. Dynin, Inversion problem for singular integral operators: $C^{*}$-approach, Proc. Nat. Acad. Sci. U.S.A. 75 (1978), 4668-4670.

6. G. G. Kasparov, The operator $K$-functor and extensions of $C^{*}$-algebras, Izv. Akad. Nauk SSSR Ser. Mat. 44 (1980), 571-636.

7. O. Loos, Bounded symmetric domains and Jordan pairs, Univ. of California, Irvine, 1977.

8. W. Schmid, Die Randwerte holomorpher Funktionen auf hermitesch symmetrischen Räumen, Invent. Math. 9 (1969), 61-80.

9. H. Upmeier, Toeplitz operators on bounded symmetric domains, Trans. Amer. Math. Soc. 280 (1983), 221-237.

10. , , Toeplitz $C^{*}$-algebras on bounded symmetric domains, Ann. of Math. (2) 119 (1984), 549-576.

11. _ Generalized Fredholm indices for Toeplitz operators on symmetric domains, preprint.

12. U. Venugopalkrishna, Fredholm operators associated with strongly pseudoconvex domains, J. Funct. Anal. 9 (1972), 349-373.

DEPARTMENT OF MATHEMATICS, UNIVERSITY OF KANSAS, LAWRENCE, KANSAS 